\title{
La clase invertida: el papel de las actividades previas en el rendimiento final del alumno
}

\author{
López-Crespo, G. ${ }^{\text {, }}$ Fidalgo, C., Martín-Albo, J. ${ }^{\mathbf{a}}$, Valdivia-Salas, S. ${ }^{\mathbf{a}}$, Lerma-Cabrera, J.M. ${ }^{\text {, }}$ \\ Carvajal-Ruiz, M. ${ }^{\mathbf{b}}$ y Carralero-Esteban, D. ${ }^{\mathbf{a}}$

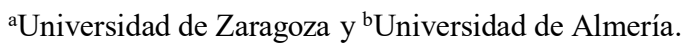

\begin{abstract}
The flipped classroom model consists on a re-arrangement of the traditional instructional model, in the sense that the lectures are placed out of the classroom whereas the classroom time is devoted to promote a more active and deeper learning in the students. This innovation was aimed to study the impact of viewing videos with questions embeded along it on the final achievent of the students. Results showed that there is a marginal relationship between watching videos and the score on the final test of the course. In addition, a possitive correlation between the scores obtained on the questions of the videos and the score on the final test was obtained. This results are in accordance with the idea that embeding questions along the videos leads to a more profound proccessing of the information, and therefore, a better learning.
\end{abstract}

Keywords: flipped-classroom, on-line videos, testing effect, academic achievement.

\section{Resumen}

El modelo de la clase invertida o flipped classroom consiste en dar la vuelta a la organización del modelo tradicional docente, sacando la transmisión de conocimientos fuera del aula y dejar tiempo de aula para propiciar un aprendizaje más activo y profundo en el alumnado. En la presente innovación se estudió el impacto que tiene visualizar vídeos con preguntas incrustadas sobre el rendimiento final en la asignatura. Los resultados mostraron por un lado que existe una relación marginalmente significativa entre la visualización de vídeos y el rendimiento en la prueba final de la asingatura. Por otro, se halló una correlación positiva aunque moderada entre las puntuaciones obtenidas en las preguntas incrustadas en los vídeos y la puntuación en el examen final. Estos resultados concuerdan con la idea de que es posible lograr un procesamiento más profundo de la información y, por tanto, un mejor recuerdo de la misma, cuando se insertan preguntas a lo largo de los vídeos.

Palabras clave: clase invertida, vídeos on-line, efecto test, rendimiento académico.

\section{Introducción}

El modelo de la clase invertida oflipped classroom, como se le conoce en inglés, es un modelo pedagógico que invierte la organización estándar del modelo instruccional tradicional. Esto es, en el modelo tradicional, el tiempo de la clase presencial se dedica a la transmisión de conocimiento (clases magistrales) y se dejan las actividades de aplicación o profundización en estos conocimientos para fuera del tiempo de aula. Sin 
embargo, en la clase invertida las actividades de transmisión del conocimiento se sacan fuera del aula, dedicándose el tiempo de aula a realizar actividades más aplicadas o de profundización (véase Figura 1).

Tabla 1. Estructuración de actividades en el modelo tradicional vs. clase invertida

ACTIVIDADES EN EL AULA

\section{MODELO TRADICIONAL MODELO INVERTIDO}

\author{
Transmisión de conocimientos \\ Aplicación de conocimientos
}

\section{ACTIVIDADES FUERA DEL AULA}

Aplicación de conocimientos

Transmisión de conocimientos

Esta inversión en cuanto a actividades de aprendizaje se traduce habitualmente en una secuencia que comienza con las actividades previas a la clase presencial (revisión de lecturas, vídeos, etc. que el estudiante realiza en el tiempo y lugar que considere), seguidas por las actividades de clase (resolución de problemas, profundización en contenidos, etc.) (DeLozier, 2016). Para las actividades de clase se suelen emplear métodos basados en la interacción entre estudiantes (Bishop, 2013); por ejemplo, se puede emplear el aprendizaje cooperativo, metodología que cuenta con una amplia evidencia a favor (Bowen, 2000; Springer, 1999).

Aunque no se trata de un modelo novedoso, es cierto que ha experimentado una gran expansión en los últimos años debido al avance y popularización de las Tecnologías de Información y Comunicación (TICs) de bajo coste (Bishop, 2013). En este sentido, las actividades previas a día de hoy consisten mayoritariamente en la visualización de vídeos.

Los defensores del modelo de la clase invertida argumentan que se trata de un modelo que permite un aprendizaje más personalizado y flexible, así como más profundo en tanto que se pueden realizar actividades más atractivas para los estudiantes (Ferrero, 2020). De acuerdo con DeLozier (2016), lo más importante es que se trata de un modelo de aprendizaje centrado en el alumno, ya que dota a este de una gran responsabilidad y autonomía en su proceso de aprendizaje, siendo el responsable de revisar el contenido a trabajar por su cuenta y a su ritmo antes de acudir a clase.

La investigación llevada a cabo muestra la eficacia de este modelo (ver por ejemplo la revisión sistemática llevadas a cabo por Chen en 2018 en el ámbito de la educación superior), si bien con ciertos matices (Ferrero, 2020).

Si bien el modelo de la clase invertida se relaciona frecuentemente con la mera visualización de vídeos, los expertos en el tema argumentan que la clase invertida es eficaz en tanto en cuanto lo sean las actividades de clase, ya que es en ellas dónde se pueden llevar a cabo actividades centradas en el alumno que incluyen un aprendizaje más activo, aprendizaje entre iguales y aprendizaje cooperativo (Akçayir, 2018). Más aún, DeLozier (2016) argumentan que el modelo es exitoso cuando se ponen en marcha procesos cognitivos de alto nivel.

Sin dejar de estar de acuerdo con esta premisa, también es cierto que se puede elevar las demandas cognitivas de una tarea basada en la transmisión de conocimientos como son los vídeos explicativos. Por ejemplo, se pueden insertar preguntas a lo largo de los vídeos, no solo para forzar a los alumnos a que estén más atentos al vídeo, sino porque de por sí realizar pruebas de evaluación o cualquier otro tipo de actividad que fuerce la recuperación de los contenidos en la memoria produce un aprendizaje más profundo y duradero. Así lo muestran numerosos resultados de investigación acerca del conocido como efecto test (testing effect) o práctica de recuperación (ver por ejemplo Roediger III, 2011).

(cc) EY-NC-ND 2020, Universitat Politècnica de València 
Si bien hasta hace unos años resultaba complicado disponer del software necesario para incrustar preguntas a lo largo de un vídeo, hoy en día contamos con esa posibilidad gracias al desarrollo de aplicaciones educativas como Edpuzzle. Edpuzzle es una aplicación gratuita, al menos en su versión básica, que permite editar vídeos ya sean de edición propia o procedentes de otras plataformas, así como revisar el cumplimiento de las actividades por parte del alumno. Entre sus funcionalidades destaca la posibilidad de incrustar preguntas a lo largo del vídeo, lo que permite al profesor, por un lado, chequear la comprensión de los contenidos del vídeo por parte de los alumnos, y, por otro, activar procesos cognitivos de más alto nivel en éstos últimos, tales como los derivados de la recuperación de la información en la memoria (testing effect). Precisamente esta aplicación se empleó en el siguiente trabajo, ya que perseguíamos conocer si la visualización de vídeos con preguntas incrustadas a lo largo del mismo se relaciona con un mayor rendimiento en la asignatura de Psicología de la Educación en el Grado de Magisterio en Educación Primaria del Campus de Teruel (Universidad de Zaragoza).

\section{Objetivos}

Habida cuenta de los antecedentes hasta ahora planteados, el objetivo de este trabajo fue comprobar si la visualización de vídeos online con preguntas incrustadas a lo largo de los mismos se traduce en un mejor rendimiento en el examen final de la asignatura. En concreto, se perseguía:

- Comprobar si existe relación entre la visualización de vídeos y el hecho de aprobar o no la asignatura

- Comprobar si la puntuación obtenida en los vídeos se relaciona con la puntuación obtenida en la prueba final de la asignatura

\section{Desarrollo de la innovación}

\subsection{Participantes}

Participaron en la experiencia un total de 115 alumnos matriculados en la asignatura de Psicología de la Educación de primero de Magisterio en Educación Primaria del Campus de Teruel. Se trata de una asignatura de primer curso y cuatrimestre.

\subsection{Procedimiento}

La innovación consistió en aplicar una secuencia típica de la clase invertida de actividades previas a las actividades de clase.

Para llevar a cabo las actividades previas el equipo docente preparó una serie de vídeos explicativos de los principales tópicos de la asignatura. Los vídeos fueron grabados partiendo de presentaciones de power point a las que se les añadió una pista de audio y se guardó con formato Windows media. Una vez grabados los vídeos se subieron a la plataforma Edpuzzle. Se trata de una sencilla plataforma de edición de vídeos que permite funcionalidades muy básicas como acortar los vídeos, insertar audios o insertar preguntas a lo largo de los mismos. En concreto, se utilizó esta última funcionalidad, por lo que a lo largo de los vídeos se insertaron unas cinco preguntas de alternativa múltiple que el alumno había de responder para poder seguir visualizando el vídeo. Para evitar picarescas se habilitó la opción de no permitir pasar el vídeo hacia delante (aunque sí se podía pasar hacia atrás las veces que lo necesitase el alumno). 
Con unos días de antelación a la clase presencial se distribuía el vídeo en cuestión a los alumnos a través de un enlace en Moodle. Su tarea consistía en ver el vídeo y responder a las preguntas que se le planteaban antes de una fecha y hora límites.

Antes de acudir a clase, la profesora revisaba el listado de alumnos que habían visualizado el vídeo y el porcentaje global de aciertos para cada pregunta, para detectar dónde encuentran los alumnos las principales dificultades.

Una vez en clase, se resolvían las dudas planteadas por los alumnos y se incidían en las cuestiones en las que se detectaban dificultades. Posteriormente, los alumnos trabajaban en grupo resolviendo ejercicios, problemas y casos relacionados con el vídeo visto antes de acudir a clase. Por último, la profesora recogía los ejercicios y los resolvía en clase.

\section{Resultados}

Para evaluar si la visualización de vídeos con preguntas incrustadas producía un mejor rendimiento en la asignatura se siguieron dos estrategias. Por un lado, se analizó mediante una prueba chi-cuadrado si existe relación entre el número de vídeos visualizados por los alumnos y el rendimiento en la prueba final de la asignatura. Por otra parte, se realizó un análisis de correlación entre las puntuaciones obtenidas en las preguntas incrustadas en los vídeos y las obtenidas en la prueba final de la asignatura. Dado que esta última tiene un carácter teórico-práctico, se realizó un análisis de correlación con las puntuaciones obtenidas en prácticas para ver si esta correlación era mayor o menor que la obtenida en los vídeos.

Respecto al primer análisis, hemos de decir que una de las dificultades que nos encontramos en esta innovación fue que los alumnos realizasen las actividades previas. Es esta una de las mayores dificultades que reportan los estudios llevados a cabo al respecto (López-Crespo, 2020). Pero, además, la asignatura cuenta con una dificultad añadida: que algunos estudiantes se matriculan en la misma una vez comenzado el curso (incluso en algunos casos hasta en noviembre). Por ello nos encontramos que, a diferencia de otras experiencias llevadas a cabo por el mismo equipo docente (López-Crespo, 2019), el porcentaje de alumnos que visualiza los vídeos es relativamente bajo.

Por este motivo se llevaron a cabo dos análisis chi-cuadrado. En el primero se analizó si existía relación entre ver la mitad de los vídeos y el rendimiento en la asignatura; en el segundo se repitió el mismo análisis, pero examinando la relación entre ver más del $95 \%$ de los vídeos y el rendimiento en la asignatura.

Respecto al primer análisis, el valor de chi-cuadrado fue de $3.31(p=.06)$. Resultados muy similares se obtuvieron en el segundo análisis, obteniéndose valores de chi-cuadrado de $2.83(p=.09)$. Por tanto, existe una relación marginalmente significativa entre ver el 50 o 90 por ciento de los vídeos con la puntuación obtenida en el examen final de la asignatura.

Respecto a los análisis de correlación, se obtuvo una relación moderada, aunque estadísticamente significativa, entre la puntuación obtenida en las preguntas incrustadas en los vídeos y las puntuaciones obtenidas en el examen final $(\mathrm{R}=.43, p .00001)$; se halló asimismo una relación débil pero estadísticamente significativa entre las puntuaciones obtenidas en las actividades prácticas de la asignatura y las puntuaciones obtenidas en el examen final $(\mathrm{R}=.24, p<.01)$.

\section{Discusión y conclusiones}

El objetivo de este trabajo fue comprobar si la visualización de vídeos con preguntas incrustadas antes de la asistencia a clase está asociado a un mejor rendimiento en la prueba final de la asignatura. Los resultados mostraron que existe una relación marginalmente significativa entre estas dos variables para aquellos

(c)) EY-NC-ND 2020, Universitat Politècnica de València 
alumnos que vieron la mitad de los vídeos; curiosamente, se halló esta misma relación en el caso de aquellos alumnos que visualizaron más del $90 \%$ de los vídeos. En contra de lo esperado, no hallamos una mayor relación cuanto mayor es el número de vídeos visualizados por los alumnos, lo que nos hace pensar que hay otras variables que están a la base de estos resultados; por ejemplo, dado que el modelo de la clase invertida es novedoso es posible que los alumnos que no son regulares viendo los vídeos sí acudan a clase de forma regular, compensando de esta manera el aprendizaje. Por otra parte, una variable no controlada en este estudio es las veces que los alumnos re-visualizan un vídeo, opción que está disponible en Edpuzzle y que nuestros alumnos emplean frecuentemente a la hora de repasar (López-Crespo et al., 2020). Esto haría que se diluyesen las diferencias entre ver el 50 o el $90 \%$ de los vídeos.

Por otra parte, los resultados muestran una correlación entre la puntuación obtenida en los vídeos con la nota final en el examen; correlación más alta que la obtenida entre la nota de prácticas y la puntuación final en el examen. Es importante remarcar en este caso que el examen es de carácter teórico-práctico, con pocas preguntas de memorización de contenidos y sí muchas de aplicación. Estos resultados son coherentes con la idea de que no es tan relevante cómo se transmite el conocimiento o si este se ajusta al esquema de la clase invertida o no, sino la calidad de los procesos cognitivos que se ponen en marcha (DeLozier, 2016). Aquellos alumnos que sacan mayores puntuaciones en la visualización de vídeos son probablemente los que hayan puesto en marcha procesos cognitivos de mayor profundidad (por ejemplo, esforzándose por rememorar la información), lo que produciría un mejor aprendizaje.

No obstante, futuros estudios habrán de confirmar estas afirmaciones, ya que la presente investigación no está exenta de cuestiones a mejorar. Por un lado, sería conveniente introducir un grupo control en el que no se insertasen preguntas, y comparar si el rendimiento es mejor en el grupo experimental (vídeo + preguntas) que en el grupo control (sólo vídeo). Por otro lado, habría que controlar el número de veces que los alumnos revisan los vídeos. Por último, sería interesante controlar otras variables relacionadas con las actividades de clase (asistencia, actividades realizadas en clase y calidad de las mismas) para poder dar una visión más en profundidad de qué factores contribuyen en mayor medida a la eficacia de la clase invertida.

En conclusión, los resultados aquí mostrados parecen indicar que visualizar los vídeos en el modelo de la clase invertida se asocia, aunque débilmente, con un mejor rendimiento en la prueba final de la asignatura, y que la puntuación obtenida en las preguntas incrustadas se relaciona positivamente con un mayor rendimiento en dicha prueba.

\section{Referencias}

AKÇAYIR, G., Y AKÇAYIR, M. (2018). "The flipped classroom: A review of its advantages and challenges" en Computers \& Education, 126, 334-345.

BISHOP, J. L., \& VERLEGER, M. A. (2013). "The flipped classroom: A survey of the research". En ASEE National Conference Proceedings, Atlanta, GA, , 30(9) 1-18.

BOWEN, C. W. (2000). "A quantitative literature review of cooperative learning effects on high school and college chemistry achievement". En Journal of Chemical Education, 77(1), 116.

CHEN, K., MONROUXE, L., LU, Y., JENQ, C., CHANG, Y., CHANG, Y., \& CHAI, P. Y. (2018). "Academic outcomes of flipped classroom learning: A meta-analysis". En Medical Education, 52(9), 910-924. doi:10.1111/medu. 13616

DELOZIER, S. J., \& RHODES, M. G. (2017). "Flipped classrooms: A review of key ideas and recommendations for practice". En Educational Psychology Review, 29(1), 141-151. 
FERRERO, M. (2020). ¿Qué dice la investigación sobre el aula invertida?

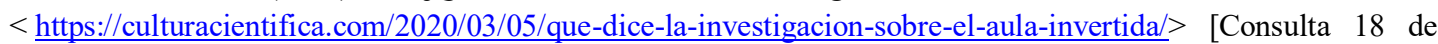
marzo de 2020]

LÓPEZ-CRESPO, G., FIDALGO, C., LERMA-CABRERA, J.M. Y CARRALERO-ESTEBAN, D. (2020). "Elaboración de vídeos docentes: más allá de la clase invertida". En Innovagogía 2020.

LÓPEZ-CRESPO, G., MARTÍN-ALBO, J., VALDIVIA-SALAS, S., Y CARRALERO-ESTEBAN, D. (2019). "Potenciando el papel activo del alumnado a través de la clase invertida: Análisis de las actividades de clase, metacognición y rendimiento académico". En Allueva Pinilla, A.I. y Alejandre Marco, J.L. ACTAS del congreso internacional virtual USATIC 2019, ubicuo y social: Aprendizaje con TIC. Zaragoza: Prensas de la Universidad de Zaragoza.

ROEDIGER III, H. L., \& BUTLER, A. C. (2011). "The critical role of retrieval practice in long-term retention". En Trends in Cognitive Sciences, 15(1), 20-27.

SPRINGER, L., STANNE, M. E., \& DONOVAN, S. S. (1999). "Effects of small-group learning on undergraduates in science, mathematics, engineering, and technology: A meta-analysis". En Review of Educational Research, 69(1), 21-51. doi:10.3102/00346543069001021 